\title{
A Case Report and Review of Postural Orthostatic Tachycardia Syndrome in Pregnancy
}

\author{
Brianna Lide, $\mathrm{BA}^{1} \quad$ Sina Haeri, MD, MHSA ${ }^{2,3}$ \\ ${ }^{1}$ Texas A and M University College of Medicine, Temple, Texas \\ ${ }^{2}$ St. David's North Austin Medical Center, Austin, Texas \\ ${ }^{3}$ Department of Obstetrics and Gynecology, Baylor College of \\ Medicine, Houston, Texas
}

\begin{abstract}
Address for correspondence Sina Haeri, MD, MHSA, North Austin Maternal-Fetal Medicine, 12200 Renfert Way, Suite G-3, Austin, TX 78758 (e-mail: SinaHaeri@gmail.com).
\end{abstract}

Am J Perinatol Rep 2015;5:e33-e36.

\begin{abstract}
Keywords

- pregnancy

- postural orthostatic tachycardia

- POTS

- syndrome

Purpose Postural orthostatic tachycardia syndrome (POTS) is a form of orthostatic intolerance characterized by an increased heart rate upon transition from supine to standing, and head-up tilt without orthostatic hypotension. Its etiology is multifactorial, and no clear cause has been identified. Common symptoms include light-headedness, blurred vision, weakness, cognitive difficulties, and fatigue and are often accompanied by palpitations, shortness of breath, syncope, or gastrointestinal symptoms. Management includes volume expansion, physical counter maneuvers, and pharmacological agents such as fludrocortisone, midodrine, propranolol, and pyridostigmine. The course of POTS in pregnancy is variable and POTS has not been directly implicated in any adverse outcomes for the mother or fetus.

Methods Two cases of POTS in pregnancy are presented, along with a review of the literature for reports of POTS in pregnancy.

Results Along with our 2 cases, 10 other case reports were identified and included. Conclusion The course of POTS in pregnancy is variable, and not directly linked to increase perinatal morbidity or mortality. Women can safely undergo regional anesthesia, and vaginal delivery with close monitoring of hemodynamic changes.
\end{abstract}

Postural orthostatic tachycardia syndrome, POTS, is an autonomic condition resulting in a marked increase in heart rate ( $\geq 30$ beats per minute) or a tachycardia ( $\geq 120$ beats per minute), upon standing or tilt table testing without orthostatic hypotension. It is more frequent in females, (female: male ratio of 4.5:1), and most cases occur between ages of 15 and 25 years. ${ }^{1}$ The tachycardia response may be caused by inefficient sympathetic vasoconstriction of the lower limbs, excessive sympathetic drive, volume dysregulation, and physical deconditioning. Common exacerbating factors include excessive heat exposure, physical exertion, anxiety, depression, and prolonged bed rest. ${ }^{1}$

A current review of the literature demonstrates that the course of POTS in pregnancy is variable with similar proportions of patients reporting unchanged, worsened, and improved symptoms. ${ }^{2-5}$ For those patients who did report worsening symptoms, hyperemesis gravidarum, ${ }^{2,6} \mathrm{mi}-$ graine, ${ }^{3}$ and presyncopal and syncopal episodes ${ }^{4,7}$ were most common. The majority of studies have concluded that POTS does not appear to contribute to pregnancy-related complications s,4,8 $^{3,4}$ show that vaginal delivery and regional anesthesia are generally safe in women with POTS.

\section{Case Description}

\section{Case 1}

A 34-year-old Caucasian woman, gravida 2, para 1,001, was referred at 9 weeks to our Maternal-Fetal Medicine clinic for a pregnancy complicated by POTS (physical deconditioning type). Her medical history was significant only for the POTS, which was diagnosed after extensive evaluation 4 years earlier. She had previously been well managed with conservative measures, received

July 16, 2014 accepted after revision

November 28, 2014

published online

March 4, 2015
DOI http://dx.doi.org/ 10.1055/s-0034-1544106. ISSN 2157-6998.
Copyright $\odot 2015$ by Thieme Medical Publishers, Inc., 333 Seventh Avenue, New York, NY 10001, USA. Tel: +1(212) 584-4662.
License terms

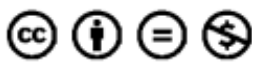


although with her prior pregnancy she did require $\beta$-blockade. The pregnancy had resulted in an uncomplicated delivery of a healthy and normally grown fetus at 38 weeks gestation. Although initially unremarkable without pharmacotherapy, she did experience disease exacerbation at approximately 15 weeks, which was not amenable to conservative measures. Propranolol ( $40 \mathrm{mg}$, twice daily) was started, which resulted in resolution of her symptoms. The remainder of the pregnancy was uncomplicated and resulted in a 40 -week spontaneous labor and vaginal delivery of a healthy 3,638 $\mathrm{g}$ male infant with Apgar scores of 9 and 9 . She did receive regional anesthesia (epidural) without complication. As of 6 weeks postpartum, she had an uneventful course and was doing well with on-going propranolol therapy ( $40 \mathrm{mg}$, twice daily).

\section{Case 2}

A 26-year-old Caucasian woman, gravida 1, para 0, was referred at 21 weeks to our Maternal-Fetal Medicine clinic for a pregnancy complicated by POTS (physical deconditioning type). Her medical history was significant only for the POTS, which was diagnosed 2 years earlier and managed by her Neurologist. She had previously been well managed with conservative measures, although exacerbation in the summer had required successful treatment with fludrocortisone. She had been started on propranolol ( $80 \mathrm{mg}$, once daily) by Neurology at 10 weeks due to exacerbation, which they had attributed to her underlying hyperemesis gravidarum. She continued propranolol ( $80 \mathrm{mg}$, once daily) throughout the pregnancy, which resulted in a 38-week spontaneous labor and vaginal delivery of a healthy 3,229 $\mathrm{g}$ female infant with Apgar scores of 8 and 9. She did receive regional anesthesia (epidural) without complication. As of 6 weeks postpartum, she had an uneventful course and was doing well with on-going propranolol therapy.

\section{Discussion}

\section{Presentation and Diagnosis}

The diagnosis of POTS in pregnancy is difficult, as pregnancy itself may induce similar symptoms. ${ }^{5}$ Diagnosis is made by careful history and the presence of a sustained heart rate increase by $\geq 30$ beats per minute upon the transition from supine to standing position, improving in the recumbent position, for 6 months duration or longer. A standing heart rate of $\geq 120$ beats per minute may also indicate POTS, but this may not be an appropriate criterion for those with a low resting rate. A standing norepinephrine level $\geq 600 \mathrm{pg} / \mathrm{mL}$ (seen in $30-60 \%$ of POTS patients ${ }^{1}$ ) and other clinically indicated laboratory, cardiac, and neurologic evaluations can be used to confirm diagnosis. ${ }^{1,4}$ However, other causes of orthostatic symptoms should be ruled out before extensive cardiac or neurologic testing is performed, and may include thyroid dysfunction, pregnancy, anemia, diabetes, and more. ${ }^{4}$

\section{Classification}

There are several subtypes of POTS, including neuropathic, hyperadrenergic, and POTS-related to volume dysregulation and physical deconditioning. Knowing a patient's POTS subtype is important in directing management of this condition in pregnancy and delivery. ${ }^{9}$ Neuropathic POTS describes patients who demonstrate a loss of peripheral sympathetic innervation to the lower limbs resulting in venous pooling. Hyperadrenergic POTS is caused by excessive sympathetic drive and is marked by a standing plasma norepinephrine $\geq 600 \mathrm{pg} / \mathrm{mL}$, and fluctuating and/or increased blood pressure during head-up tilt. In patients with hyperadrenergic POTS, episodes may be triggered by emotional and physical stimuli. Patients with the volume dysregulation variant of POTS have low plasma, red cell, and total blood volumes. This could be related to low plasma renin and aldosterone activity, high plasma angiotensin II levels indicating reduced ACE-2 activity, and gastrointestinal disorders resulting in poor fluid intake and/or retention. Physical deconditioning is another form of POTS, and is revealed by significant and persistent tachycardia during exercise or when upright, as well as reduced stroke volume and a relatively low peak oxygen uptake compared with control groups. This could be caused by an inefficient baroreceptor reflex and vestibulosympathetic reflex due to bed rest or deconditioning. ${ }^{1}$

\section{Antepartum Considerations}

The course of POTS in pregnancy is variable. ${ }^{2-5}$ In general, patients who did not require treatment before pregnancy were less likely to report exacerbation during pregnancy. ${ }^{4}$ One retrospective case study performed by Khalil et al, evaluating outcomes of 22 pregnant women with preexisting POTS, reported the worsening of symptoms occurred solely in the first trimester, and the authors attributed symptom resolution thereafter to increased fluid retention during the second and third trimesters. ${ }^{3}$ However, a case review by Glatter et al of two other patients with severe POTS reported a worsening of symptoms at 6 months gestation, and hyperemesis gravidarum. ${ }^{6}$ Kimpinski et al compared POTS in parous and nulliparous women looking at POTS in 116 pregnancies and found no notable difference in the course of the condition between pregnant women, and women who were not pregnant. ${ }^{8}$ Peggs et al conducted a similar study of 29 pregnant women with POTS and found no difference in symptoms of faintness before and during pregnancy. ${ }^{10}$

The majority of studies have concluded that POTS does not appear to contribute to pregnancy-related complications. ${ }^{3,4,8,10}$ A retrospective study by Blitshteyn et al, examining 10 women with a total of 17 live births reported a higher miscarriage rate in this study cohort ( $41 \%$, excluding a strong outlier), which is higher than the rate of the general population (31\%). ${ }^{2}$ This finding was contrary to that of other studies of women with preexisting POTS. ${ }^{3-6,8}$

\section{Intrapartum Considerations}

Many women with POTS have been able to undergo safe vaginal delivery, ${ }^{3-5,7,11}$ even without anesthesia. ${ }^{4}$ The presence of POTS should generally not influence the choice of whether to deliver vaginally or by cesarean section. In some cases it may be important to establish analgesia early 
in labor to prevent a tachycardic response. ${ }^{7,9}$ It has been shown that nonpregnant patients with POTS experience a relatively larger decrease in blood pressure in the early phases of Valsalva, accompanied by a subsequently large increase in blood pressure and heart rate in the later phases. McEvoy et al, who examined the anesthetic management of POTS in delivery, suggested that in the pregnant patient these hemodynamic changes could increase pelvic blood vessel resistance thereby decreasing uteroplacental blood flow. They therefore concluded that it is safest to monitor hemodynamics frequently especially in the second phase of labor and consider avoiding Valsalva by opting for instrumental or cesarean delivery if hemodynamic variations do not return to normal between contractions. ${ }^{9}$ It must be cautioned that this is the only study to suggest an alteration in the mode of delivery.

\section{Therapy}

Management of POTS includes volume expansion, physical counter maneuvers, pharmacotherapy, and behavioralcognitive therapy. Most patients require volume expansion with adequate intake of water and sodium. Caffeine intake should be limited as it may promote hypovolemia by increasing diuresis. Physical counter maneuvers may be helpful by producing small increases in mean arterial pressure and include making a fist, bending forward, and placing a foot on a chair. Compression stockings may also be helpful. ${ }^{1}$

Pharmacologic treatments include mineralocorticoids such as fludrocortisone, $\alpha-1$ agonists such as midodrine, $\beta$ blockers such as propanolol, and cholinesterase inhibitors such as pyridostigmine. ${ }^{1}$ Below is a brief overview of pregnancy and lactation consideration for the four more commonly employed pharmacotherapies for POTS:

1. Fludrocortisone: There are no pregnancy or lactation studies on fludrocortisone specifically; however, the data with respect to glucocorticoid use in pregnancy suggest an increased risk for cleft palate and possible impaired fetal growth and an otherwise relatively safe profile. ${ }^{12}$ The American Academy of Pediatrics classifies glucocorticoids as compatible with breastfeeding. ${ }^{13}$

2. Midodrine: Pregnancy data with midodrine is limited to the case report by Glatter et $\mathrm{al}^{6}{ }^{6}$ which included a normal infant. There are otherwise no human data on pregnancy or lactation with respect to this agent. In general, due to its vasoconstrictive properties, it should be used with caution due to theoretically increased risk for fetal vascular insults (e.g., gastroschisis). ${ }^{14}$

3. Propranolol: Pregnancy data with respect to propranolol have not indicated an increased risk for teratogenicity; however, an association with neonatal apnea, respiratory distress, bradycardia, and hypoglycemia has been suggested. ${ }^{15}$ The American Academy of Pediatrics classifies it as compatible with breastfeeding. ${ }^{13}$

4. Pyridostigmine: Pregnancy data have not indicated an increased risk for teratogenicity or adverse perinatal outcomes. ${ }^{16}$ The World Health Organization and American
Academy of Pediatrics classify pyridostigmine as compatible with breastfeeding. ${ }^{13,17}$

5. Phenylephrine is commonly used to treat hypotension associated with neuraxial blockade in POTS patients. ${ }^{7,9}$ Although a vasoconstricting agent (similar to midodrine), teratogenicity, and lactation are not a major concern, as this agent is generally used intrapartum and in a short exposure window.

In conclusion and based on our cases and review of the literature, POTS does not appear to confer an increased risk of adverse perinatal outcomes. While the first trimester may confer increased risk of disease exacerbation, the fluid retention in second and third trimester should prove beneficial. Management should start with identification of the subtype of POTS, and include conservative measures before initiation of pharmacotherapy. Propranolol should be the first considered agent due to its safe pregnancy and lactation profile. Timing and mode of delivery should be reserved for usual obstetrical indications.

Conflict of Interest

Authors state that there is no competing interest or financial disclosure for this article.

\section{References}

1 Benarroch EE. Postural tachycardia syndrome: a heterogeneous and multifactorial disorder. Mayo Clin Proc 2012;87(12): 1214-1225

2 Blitshteyn S, Poya H, Bett GCL. Pregnancy in postural tachycardia syndrome: clinical course and maternal and fetal outcomes. J Matern Fetal Neonatal Med 2012;25(9):1631-1634

3 Kanjwal K, Karabin B, Kanjwal Y, Grubb BP. Outcomes of pregnancy in patients with preexisting postural tachycardia syndrome. Pacing Clin Electrophysiol 2009;32(8):1000-1003

4 Powless CA, Harms RW, Watson WJ. Postural tachycardia syndrome complicating pregnancy. J Matern Fetal Neonatal Med 2010;23(8):850-853

5 Pramya N, Puliyathinkal S, Sagili H, Jayalaksmi D, Reddi Rani P. Postural orthostatic tachycardia syndrome complicating pregnancy: a case report with review of literature. Obstet Med 2012;5(2): 83-85

6 Glatter KA, Tuteja D, Chiamvimonvat N, Hamdan M, Park JK. Pregnancy in postural orthostatic tachycardia syndrome. Pacing Clin Electrophysiol 2005;28(6):591-593

7 Corbett WL, Reiter CM, Schultz JR, Kanter RJ, Habib AS. Anaesthetic management of a parturient with the postural orthostatic tachycardia syndrome: a case report. Br J Anaesth 2006;97(2): 196-199

8 Kimpinski K, Iodice V, Sandroni P, Low PA. Effect of pregnancy on postural tachycardia syndrome. Mayo Clin Proc 2010;85(7): 639-644

9 McEvoy MD, Low PA, Hebbar L. Postural orthostatic tachycardia syndrome: anesthetic implications in the obstetric patient. Anesth Analg 2007;104(1):166-167

10 Peggs KJ, Nguyen H, Enayat D, Keller NR, Al-Hendy A, Raj SR. Gynecologic disorders and menstrual cycle lightheadedness in postural tachycardia syndrome. Int J Gynaecol Obstet 2012; 118(3):242-246 
11 Kodakkattil S, Das S. Pregnancy in woman with postural orthostatic tachycardia syndrome (POTS). J Obstet Gynaecol 2009;29(8): 764-765

12 Park-Wyllie L, Mazzotta P, Pastuszak A, et al. Birth defects after maternal exposure to corticosteroids: prospective cohort study and meta-analysis of epidemiological studies. Teratology 2000; 62(6):385-392

13 American Academy of Pediatrics Committee on Drugs. Transfer of drugs and other chemicals into human milk. Pediatrics 2001; 108(3):776-789
14 Torfs CP, Katz EA, Bateson TF, Lam PK, Curry CJR. Maternal medications and environmental exposures as risk factors for gastroschisis. Teratology 1996;54(2):84-92

15 Campbell JW. A possible teratogenic effect of propranolol. N Engl J Med 1985;313(8):518

16 Heinonen OP, Slone D, Shapiro S. Birth defects and drugs in pregnancy. In: Slone D, ed. Littleton, MA: Publishing Sciences Group; 1977:345-356

17 Bennett PN. Drugs and Human Lactation. Amsterdam: Elsevier Science Publishers; 1988:402-403 